OPEN ACCESS

Edited by:

Richard D. Boyle,

National Aeronautics and Space Administration (NASA), United States

Reviewed by:

Danilo Cialoni,

Dan Europe Foundation, Italy Jacek Kot,

Medical University of Gdańsk, Poland Rodrigue Pignel,

Université de Genève, Switzerland

${ }^{*}$ Correspondence: Salih Murat Egi smegi@daneurope.org

Specialty section: This article was submitted to Environmental, Aviation and Space

Physiology,

a section of the journal

Frontiers in Physiology

Received: 03 February 2019 Accepted: 06 June 2019

Published: 11 July 2019

Citation:

Imbert J-P, Egi SM, Germonpré P and Balestra C (2019) Static

Metabolic Bubbles as Precursors of Vascular Gas Emboli During Divers' Decompression: A Hypothesis Explaining Bubbling Variability. Front. Physiol. 10:807. doi: 10.3389/fphys.2019.00807

\section{Static Metabolic Bubbles as Precursors of Vascular Gas Emboli During Divers' Decompression: A Hypothesis Explaining Bubbling Variability}

\author{
Jean-Pierre Imbert ${ }^{1}$, Salih Murat Egi' ${ }^{2,3 *}$, Peter Germonpré ${ }^{3,4}$ and Costantino Balestra ${ }^{3,5}$ \\ ${ }^{1}$ Divetech, Biot, France, ${ }^{2}$ Department of Computer Engineering, Galatasaray University, Istanbul, Turkey, ${ }^{3}$ DAN Europe \\ Research Division, Divers Alert Network (DAN), Roseto, Italy, ${ }^{4}$ Centre for Hyperbaric Oxygen Therapy, Military Hospital \\ Brussels, Brussels, Belgium, ${ }^{5}$ Environmental, Occupational and Ageing Physiology Laboratory, Haute Ecole \\ Bruxelles-Brabant (HE2B), Brussels, Belgium
}

Introduction: The risk for decompression sickness (DCS) after hyperbaric exposures (such as SCUBA diving) has been linked to the presence and quantity of vascular gas emboli (VGE) after surfacing from the dive. These VGE can be semi-quantified by ultrasound Doppler and quantified via precordial echocardiography. However, for an identical dive, VGE monitoring of divers shows variations related to individual susceptibility, and, for a same diver, dive-to-dive variations which may be influenced by pre-dive pre-conditioning. These variations are not explained by currently used algorithms. In this paper, we present a new hypothesis: individual metabolic processes, through the oxygen window $(\mathrm{OW})$ or Inherent Unsaturation of tissues, modulate the presence and volume of static metabolic bubbles (SMB) that in turn act as precursors of circulating VGE after a dive.

Methods: We derive a coherent system of assumptions to describe static gas bubbles, located on the vessel endothelium at hydrophobic sites, that would be activated during decompression and become the source of VGE. We first refer to the OW and show that it creates a local tissue unsaturation that can generate and stabilize static gas phases in the diver at the surface. We then use Non-extensive thermodynamics to derive an equilibrium equation that avoids any geometrical description. The final equation links the $\mathrm{SMB}$ volume directly to the metabolism.

Results and Discussion: Our model introduces a stable population of small gas pockets of an intermediate size between the nanobubbles nucleating on the active sites and the VGE detected in the venous blood. The resulting equation, when checked against our own previously published data and the relevant scientific literature, supports both individual variation and the induced differences observed in pre-conditioning experiments. It also explains the variability in VGE counts based on age, fitness, type and frequency of physical activities. Finally, it fits into the general scheme of the arterial bubble assumption for the description of the DCS risk. 
Conclusion: Metabolism characterization of the pre-dive SMB population opens new possibilities for decompression algorithms by considering the diver's individual susceptibility and recent history (life style, exercise) to predict the level of VGE during and after decompression.

Keywords: diving, decompression sickness, desaturation, oxygen window, pre-conditioning

\section{INTRODUCTION}

Hyperbaric exposures such as SCUBA diving are associated with a risk of decompression sickness (DCS). Traditionally, this risk has been attributed to the presence and quantity of decompression bubbles in the blood (vascular gas emboli or VGE) causing blood flow occlusion in various tissues. Recently, DCS has also been associated to the presence of blood microparticles and some symptoms linked to an inflammatory process (Thom et al., 2011; Arieli et al., 2015; Spisni et al., 2017).

In order to limit the risk of DCS, decompression procedures have been developed, as early as from 1908 (Haldane's first experiments and publication of "dive decompression tables"). Although most diving operations are still conducted with empirical models, there has been a continuous research for editing safer decompression tables based on more realistic algorithms (Hugon, 2014). Various models have been developed and tested, and some have been implemented in diving decompression computers like the Bühlmann ZHL16 algorithm.

The objective of these models is to avoid or reduce bubble formation. However, there is evidence that bubbles are present in most, if not all decompressions, without necessarily representing a threat for the diver (Papadopoulou et al., 2013, 2015). VGE have been detected during decompression by trans-thoracic ultrasonic Doppler since 35 years. These bubbles have been estimated to be of $50 \mu \mathrm{m}$ or larger size, in order to be detectable (Spencer, 1976). Graded levels of bubble detection have been used as an indication of decompression stress and, indirectly, risk of DCS (Nishi et al., 2003). A more sensitive method of monitoring is based on 2D echocardiography and uses actual bubble counts as an indication of the decompression stress (Germonpre et al., 2014). However, the resolution of the standard B-mode echography technique remains limited to roughly $35 \mu \mathrm{m}$ (Papadopoulou et al., 2014, 2017), and it is reasonable to accept that in many divers, undetectable smaller precursor bubbles are present. The variability observed in levels of VGE measured in different divers, even when performing exactly the same dive profile, suggests that these precursors play an essential role in VGE production (Papadopoulou et al., 2018).

While current technology does not allow the direct detection of the precursors of these large vascular (venous) bubbles, their existence has been proven by indirect experiments in shrimps (Evans and Walder, 1969) and rats (Vann et al., 1980). These precursors were named "gas micronuclei" and estimated to be the size of 50-100 nm (Arieli et al., 2002). Linking these micronuclei to actual VGE detected in decompressing divers is still a matter of debate and requires several steps (Blatteau et al., 2006; Doolette, 2019).
The first step deals with cavitation in physical systems. Hills showed that cavitation occurs at the liquid/liquid interface after decompression when one of the liquids is hydrophobic (Hills, 1967). Recently, Arieli established that nanobubbles form on flat hydrophobic surface of silicon wafers from dissolved gas (Arieli and Marmur, 2011) and that these nanobubbles expand and detach to form free-floating bubbles after decompression (Arieli and Marmur, 2013a; Papadopoulou et al., 2015). This establishes a link between stationary nanobubbles on the blood vessel wall and blood-borne bubbles which prepares the scenario for decompression VGE.

The second step introduces cavitation in biological systems. Hills studied various endothelial surfaces from sheep and humans for their hydrophobicity, using a method based on the angle of contact, and found distinct hydrophobic areas (Hills, 1992). He concluded that the oligolamellar surfactant lining and lamellar bodies were potentially important factors in influencing bubble formation on vessel walls. Similarly, Arieli showed that the production of bubbles after decompression of ovine blood vessels is associated to active hydrophobic spots (AHS) that stain for lipids (Arieli and Marmur, 2013b, 2014) and confirmed that these AHS consist of deposit of hydrophobic lipids similar to or even originating from lung surfactant (Arieli, 2015).

Arieli was then able to visually observe the whole dynamics of bubble growth, detachment and the rate of bubble production. He derived a mathematical equation for conditional detachment which he based on buoyancy, and therefore, on reaching a critical bubble volume (Papadopoulou et al., 2015; Arieli and Marmur, 2017). He concluded that decompression bubbles in divers can develop only from pre-existing gas micronuclei (Arieli and Marmur, 2017), and that these could be nanobubbles appearing on active hydrophobic spots (AHS) as found on the endoluminal surface of blood vessels.

Studies on diver pre-conditioning have confirmed that stable stationary bubbles are most probably already present in the diver before the dive. This pre-existing bubble population can be affected by vibrations (Germonpre et al., 2009), exercise (Dujic et al., 2004; Castagna et al., 2011), sauna (Blatteau et al., 2008), and oxygen breathing (Castagna et al., 2009) before the dive. This population can also be modified by drugs that change endothelial function (Wisloff et al., 2004). All these preconditioning protocols reduce bubble formation and/or growth as demonstrated by reduced VGE levels measured after the dive. These experiments raise two questions:

- How could a small gas phase exist and remain stable during the ordinary life of a person before he becomes involved in a dive? 
- How can factors such vibrations, exercise, heat, oxygen breathing, and endothelial-modulating drugs interfere with the bubbling process?

Recently, we showed (Balestra et al., 2016b; Germonpre and Balestra, 2017) that pre-dive vibrations better protect the diver than pre-dive oxygen breathing in terms of post-dive VGE count. We also observed that preconditioning with oxygen breathing before or during vibration is less efficient than vibration alone.

In this paper, we attempt to define a coherent system of assumptions that can:

- Describe a stable gas phase compatible with the properties of pre-existing bubbles involved in diver preconditioning experiments,

- Explain the differences observed between various pre-conditioning protocols.

For this purpose, we derive a simple mathematical model for the stability of such gas phase that provides an equilibrium equation based on local tissue metabolism. We then test the resulting equation against our previously published data (Balestra et al., 2016b) and the general literature to validate its predictions.

\section{METHODS}

Our theoretical approach is based on two identified dimensions of the problem, mechanical action related to vibrations, and metabolism, which we suspected was physically acting behind the scene through the "tissue inherent unsaturation" (Hills and LeMessurier, 1969; Hills, 1970).

To fill the gap between ex-vivo experiments and actual dives monitoring, we introduce metabolism as a parameter, characterizing any living organism. We postulate that metabolism can sustain a population of small gas pockets in the diver's tissues before he starts his dive. We give those gas pockets the name of "Static Metabolic Bubbles" or SMB.

When considering these hypothetic SMB, the first problem is the stability of such gas phases. In a stable situation, because of surface tension, there must be a pressure difference between the sum of the gas partial pressures in the gas phase and the surrounding (ambient) pressure. The source for this pressure difference must be identified and quantified because it bears implications on the size of the gas phase and its possible evolution during decompression.

The second problem is the shape of this gas phase and the computation of its interfacial energy. Laplace's law can only be used in configurations, where explicit curvatures can be defined, like for a sphere or a conical crevasse (Chappell and Payne, 2007), a characteristic that cannot be assumed for these biological gas pockets - of unknown shape. An alternative approach must be defined to account for surface tension.

\section{The Condition of Stability}

We start with a stabilization condition for the initial metabolic bubbles. Any gas phase in a tissue of a diver at the surface should contain an inert gas (nitrogen), metabolic gases (oxygen, $\mathrm{CO}_{2}$ ) and water vapor. The stability of this gas phase first depends on the dynamics of gas exchanges through its surface. Because the diffusivity of metabolic gases is high, the gas exchanges are rapid (Van Liew, 1962) and we can reasonably assume that the gases in the bubble are in equilibrium with the adjacent medium. We define the adjacent medium as the venous side of the tissue as all the studies that could analyze gas concentrations in decompression bubbles have measured values close to the venous ones (Ishiyama, 1983; Foster et al., 1998).

The rationale for the stability equation is based on the gas solubility in liquids described by Henry's law. For a dissolved gas, at equilibrium, at constant temperature:

$$
\mathrm{P}_{\mathrm{i}}=\mathrm{k}_{\mathrm{i}}^{\mathrm{H}} \mathrm{c}_{\mathrm{i}}
$$

where $P_{i}$ is the gas pressure above the liquid, $c_{i}$ the gas concentration in the liquid and $\mathrm{k}_{\mathrm{i}}^{\mathrm{H}}$, Henry's constant for the gas in the liquid at body temperature. By convention, the dissolved gas tension inside the liquid is defined as $k_{i}^{H} c_{i}$.

In the presence of a gas phase, Henry's law is written for each gas dissolved in a diver's tissue, considered to be in equilibrium with venous blood, that has diffused inside a local gas pocket. The equation below, derived by Van Liew et al. (1993), relates the gas phase pressures $(\mathrm{Pb})$ to the tissue gas tensions:

$$
\begin{aligned}
\mathrm{P}_{\mathrm{b}} & =\mathrm{P}_{\mathrm{v}, \mathrm{O}_{2}}+\mathrm{P}_{\mathrm{v}, \mathrm{CO}_{2}}+\mathrm{P}_{\mathrm{H}_{2} \mathrm{O}}+\mathrm{P}_{\mathrm{v}, \mathrm{N}_{2}} \\
& =\mathrm{k}_{\mathrm{O}_{2}}^{\mathrm{H}} \mathrm{c}_{\mathrm{O}_{2}}+\mathrm{k}_{\mathrm{CO}_{2}}^{\mathrm{H}} \mathrm{c}_{\mathrm{CO}_{2}}+\mathrm{P}_{\mathrm{H}_{2} \mathrm{O}}+\mathrm{k}_{\mathrm{N}_{2}}^{\mathrm{H}} \mathrm{c}_{\mathrm{N}_{2}}
\end{aligned}
$$

Note that because $\mathrm{CO}_{2}$ also combines into $\mathrm{HCO}_{3}^{-}$to form an acid-base buffer system that maintains the blood $\mathrm{pH}$, the $\mathrm{CO}_{2}$ concentration in the above expression only refers to dissolved molecules.

\section{The Inherent Unsaturation of Tissues}

The stability of a gas phase requires a pressure equilibrium. At this point, we hypothesize that metabolism, a purely physiological variable, could be linked to the physical world of bubbles by introducing the oxygen window (OW) as defined by Behnke (1967), also referred to as the "inherent unsaturation of tissues" by Hills and LeMessurier (1969).

The OW is a concept based on the "behavior" of metabolic gases. In a living tissue, the concentration of oxygen decreases and the dissolved $\mathrm{CO}_{2}$ concentration increases, due to metabolism. In terms of dissolved gas concentrations, the process is balanced, as there is nearly a 1 to 1 ratio between oxygen consumption and $\mathrm{CO}_{2}$ production (this is the Respiratory Quotient, varying from 1 to 0.8 at rest, depending on individual factors such as age, activity and nutrition). In terms of dissolved gas tensions, there is a significant difference as Henry's constant for $\mathrm{CO}_{2}$ is 20 times higher than the one for oxygen. The decrease in oxygen tension is much more important than the increase in $\mathrm{CO}_{2}$ tension. As a result, the sum of the gas tensions on the venous side becomes lower than on the arterial side.

This difference between the sum of the venous tissue gas tensions and the ambient pressure is called the OW. It quantifies 
the capacity of the body to take in, transport and deliver oxygen to the tissue for a given activity. Its computation involves:

- Central factors such as alveolar ventilation, respiratory quotient, cardiac output, hemoglobin concentration, $\mathrm{Hb}$ $\mathrm{O}_{2}$ affinity and perfusion rate.

- Peripheral factors such as tissue local blood flow, capillary density, oxygen extraction, oxygen diffusion and tissue metabolism.

The OW is theoretically defined by the activity of the mitochondria. Because pressures of metabolic gases are difficult to measure at this level, the $\mathrm{OW}$ is more conveniently defined across the arterial and venous sides of the tissue, for which concentrations of metabolic gases are well documented. In this work, we define the OW as the pressure difference between the ambient pressure and the tissue venous gas phase pressure as in the above-mentioned Van Liew publication:

$$
\mathrm{OW}=\mathrm{P}_{\mathrm{atm}}-\mathrm{P}_{\mathrm{b}}=\mathrm{P}_{\mathrm{atm}}-\left(\mathrm{P}_{\mathrm{v}, \mathrm{O}_{2}}+\mathrm{P}_{\mathrm{v}, \mathrm{CO}_{2}}+\mathrm{P}_{\mathrm{H}_{2} \mathrm{O}}+\mathrm{P}_{\mathrm{v}, \mathrm{N}_{2}}\right)
$$

The gas phase described in Eq. 3 is not at pressure equilibrium and must evolve toward a new state according to the second principle of thermodynamics. The ambient pressure and partial pressures of constituting gases being fixed, the only solution for the system to cope with the $\mathrm{OW}$ is to create interfacial energy.

\section{The Classic Thermodynamic Approach to Bubble Stabilization}

In Gibbs (free energy) thermodynamics, the modeling of gas supersaturated liquids requires computing the interfacial energy. Authors have proposed models, where bubbles are stabilized by Laplace's law, a skin of surfactant at the liquid/gas interface (Yount, 1979b), a diffusion barrier (Yount, 1979a), tissue elasticity (Goldman, 2010), or combinations of the aforementioned.

Using Laplace's law to express the interfacial energy between the liquid and the gas phase requires defining the curvature of the interface. For the simple case of a spherical bubble equilibrated with venous tensions it is classically expressed as:

$$
\mathrm{P}_{\mathrm{atm}}-\mathrm{P}_{\mathrm{v}}=\frac{2 \gamma}{\mathrm{r}}
$$

where $\gamma$ is the surface tension, $r$ the bubble radius and $P_{V}$ the bubble internal pressure.

For a spherical bubble attached to a flat substrate, where $\theta$ is the contact angle and $\alpha$ the base surface radius, it becomes (Yount equation):

$$
\mathrm{P}_{\mathrm{atm}}-\mathrm{P}_{\mathrm{v}}=\frac{2 \gamma \cos (\theta)}{\alpha}
$$

Explicit mathematical solutions have also been proposed for a gas phase located in a conical crevasse (Harvey et al., 1944; Tikuisis, 1986; Chappell and Payne, 2006).

In theory, for more complex configurations, splitting the interface into smaller elements of known curvatures should allow applying the Laplace's law by finite element calculations. In practice, the difficulty is to define a geometry, especially for small bubbles where the interfacial energy becomes pre-dominant. Large discrepancies have been reported between the predicted internal pressure of a nanobubble and the actual measurement in physical systems (Ohgaki et al., 2010). The differences are expected to be even worse in biological systems because of the complexity and variability of living organisms. We therefore looked for an alternative formulation of the interfacial energy, avoiding any geometrical description.

\section{The Non-extensive Thermodynamics Approach to Bubble Stabilization}

Non-extensive thermodynamics was developed to describe the behavior of solid and liquid condensates at the nanoscale, without having to refer to geometrical considerations. We chose the concept because it replaces curvature by volume and greatly simplifies our approach. It also applies without restrictions to nanometric as well as micrometric systems. The difference between the internal and external pressures of the above gas phase can be written as (Turmine et al., 2004):

$$
\mathrm{P}_{\mathrm{atm}}-\mathrm{P}_{\mathrm{b}}=\mathrm{m} \tau \alpha \frac{1}{\mathrm{~V}_{\mathrm{b}}^{1-\mathrm{m}}}
$$

where $\mathrm{V}_{\mathrm{b}}$ is the volume of gas and $\alpha$ a coefficient related to the system. The coefficient $\tau$ is an intensive variable that characterizes the interface similarly to the surface tension. It can be negative or positive which means that the system can be stabilized by either a negative or positive pressure difference, similarly to inward or outward curvature in Laplace's law. The coefficient $m$ represents the "thermodynamic dimension" of the system and is smaller than one. It somehow describes the shape of the system. For instance, setting $\tau=\gamma$ and $m=2 / 3$ allows turning the above expression back to Laplace's equation for a spherical bubble.

\section{The Static Metabolic Bubbles Volume Equation}

We combine Eqs 3 and 6 to establish the condition of stability and obtain an original equation linking the OW to the stabilization energy of the SMB.

$$
\mathrm{OW}=\mathrm{m} \tau \alpha \frac{1}{\mathrm{~V}_{\mathrm{b}}^{1-\mathrm{m}}}
$$

The above condition of stability demonstrates that:

- Metabolism can stabilize small gas pockets through the tissue inherent unsaturation;

- Metabolism controls this initial gas pockets volume.

The only requirement is a site with favorable thermodynamic conditions to generate a nanobubble that will evolve into a stable $\mathrm{SMB}$. This pre-existing SMB population in living organisms becomes a direct consequence of metabolism:

- Metabolism provides the inherent tissue unsaturation required to stabilize the SMB.

- Metabolism is a continuous process that can sustain this SMB population over time. 
- Metabolism can regenerate the SMB if the conditions are changed, provided the hydrophobic sites are persisting (Wienke and O'leary, 2018).

\section{Linking Metabolic Gas Pockets and Vascular Gas Emboli}

Once the diver enters a decompression state, the dynamic of gas exchanges will feed the SMB by diffusion from the adjacent tissue (Van Liew et al., 1993). The SMB will grow until it reaches a critical volume for bubble detachment and generate blood-borne bubbles. The level of VGE detected in the divers will therefore depend on three parameters:

(1) The density of active sites.

(2) The initial SMB volume; this will define the time it takes for an SMB to grow to its critical volume of detachment, and thus determine the delay for the first bubbles to appear.

(3) The rate of ascent that will create the diffusion gradients and define the rate at which SMB grow and VGE are produced.

\section{RESULTS}

This paper consists in developing the consequences of a mathematical hypothesis and does not bring any original experimental results. It, however, refers to a long series of experiments we conducted on divers pre-conditioning (Germonpre et al., 2009; Balestra et al., 2016b; Germonpre and Balestra, 2017) for which explanations will be presented in the discussion.

\section{Estimation of the Oxygen Window}

We calculated the theoretical OW based on Egi's model (Egi, 1994). At surface, the inherent unsaturation is estimated at $70 \mathrm{hPa}$. At depth, tissue oxygen depends on the diver's inhaled oxygen partial pressure. An increase of the $\mathrm{pO}_{2}$ in the breathing gas drastically influences the OW. This increase is linear until a point where the amount of dissolved oxygen is such that the tissue only consumes the dissolved oxygen and the hemoglobin remains maximally saturated. In this model, this point is achieved at around 2,200 hPa of $\mathrm{pO}_{2}$, which corresponds to a diver breathing pure oxygen at a depth of $12 \mathrm{~m}$. Beyond this point - which exceeds operational diving limits - the OW levels out at around 2,200-2,300 hPa (see Figure 1).

\section{Estimation of the Static Metabolic Bubbles Volume}

Following the determination of the OW, Eq. 7 theoretically permits calculating the gas phase volume. However, the coefficients used in the formula cannot be defined numerically because of the lack of experimental data. To obtain an estimation, we adapted Eq. 7 to spherical bubbles by combining Eqs 4 and 6 to obtain:

$$
\mathrm{OW}=\frac{2 \gamma}{\mathrm{r}}
$$

This way, using a surface tension of $0.050 \mathrm{~N} . \mathrm{m}^{-1}$ (Van Liew and Raychaudhuri, 1997) and two extreme values of the OW for diver breathing air at surface and a diver breathing pure oxygen at $12 \mathrm{~m}$, we calculated a bubble radius ranging from 0.45 to $14 \mu \mathrm{m}$ (Table 1).

The bubbles radii obtained are too large to be physiologically relevant. For a diver at surface, the bubble radius would correspond to 4 times the radius of a red blood cell and would not fit into a small blood capillary without seriously impairing the blood flow. This simply demonstrates that SMB are most probably not spherical and fully justifies our choice of Non-extensive thermodynamics based on volume rather than on radius.

\section{DISCUSSION}

\section{Limits to the Model: Sites for Static Metabolic Bubbles}

Equation 7 is a static equation that does not carry any information on the initial growth of the SMB. It simply defines their stability once they have formed and reached a given size. It ignores the nucleation process and growth.

Equation 7 also does not bring any indication on the location nor the geometry of the sites (crevices, flat surfaces, and hydrophobic spots). It does not exclude any possibility either. It remains compatible with gas bubbles attached to hydrophobic surfaces on the vessel endothelium as described by Arieli.

With regard to the availability of these sites, we identify three parameters to characterize a given diver at surface, prior to a dive:

(1) The SMB population depends on the number of available sites for nucleation. For consistency with Arieli's work, we

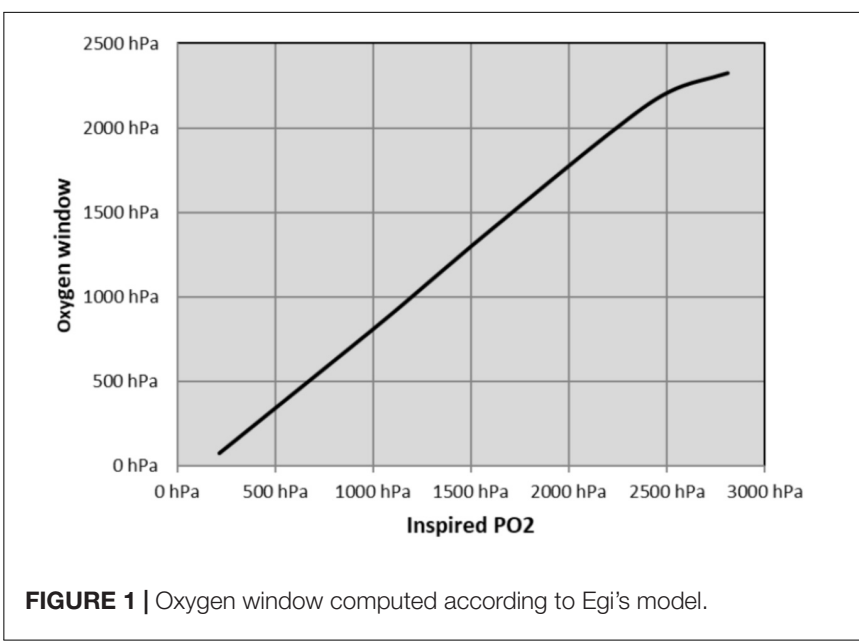

TABLE 1 | Estimated radius of spherical SMB for two diver situations.

\begin{tabular}{lcc}
\hline & OW & Spherical SMB radius \\
\hline Diver at surface breathing air & $70 \mathrm{hPa}$ & $14 \mu \mathrm{m}$ \\
Diver at $12 \mathrm{~m}$ breathing pure oxygen & $2200 \mathrm{hPa}$ & $0.45 \mu \mathrm{m}$
\end{tabular}


considered hydrophobic sites located on the blood vessel endothelium. Therefore, for a given diver, we define a first parameter corresponding to the site density, that is the number of sites available per unit of tissue volume. This site density is a long-term characteristic that may evolve with age, as does metabolism (Cialoni et al., 2017).

(2) Because all the available sites may not be populated at all times, we further define the AHS, corresponding to sites actually occupied by a SMB. The effective AHS density depends on the recent history and condition of the diver, such as pre-conditioning interventions, or several dives with sufficient surface interval.

(3) The diver is finally characterized by the volume of his SMB population. According to Eq. 7, this volume depends on the tissue metabolism. It also depends on the inspired $\mathrm{pO}_{2}$ which much influences the magnitude of the OW.

For what concerns decompression, our understanding is that SMB could be present in any place that fulfils two conditions: a favorable physical site and enough tissue super saturation. Arieli observed in his first experiments that bubbles could generate from a silicon wafer, a purely physical support (Arieli and Marmur, 2011), but this required a high gas gradient created by decompression. In his following ex-vivo experiments, the tissues had no metabolism and the bubble formation remained purely physical even if they involved real tissues as a support. In divers, our mathematical derivation of the SMB stability considers the venous side of a tissue. However, it is admitted that SMB could form as well in the lymphatic vessels (Hugon et al., 2009; Balestra, 2014b) or the distal arterial tree (Arieli and Marmur, 2017).

\section{Limit to the Model: Size of the Static Metabolic Bubbles}

The problem of identifying the gas micronuclei traditionally associated to decompression bubbles has recently been reviewed by Doolette (2019). He stressed the contradiction between the nanobubbles described in the physical chemistry literature and the physiological conditions of decompression, in particular, the extreme internal pressure calculated by Laplace's law for a small spherical bubble. If the existence of SMB still remains hypothetical, their description avoids at least two pitfalls. First, they have no defined shape, just a volume, and therefore escape Laplace's law (applicable only to a spherical bubble). Their interfacial energy can be much lower, as one could imagine when figuring for instance a tubular bubble. Secondly, their size is larger. These gas pockets are expected to have evolved to an intermediate volume set between the nanobubbles initially cavitating at the AHS and the critical volume bubbles that detach from these AHS to become VGE.

At surface, we could consider that various tissues in the diver's body will have different metabolic rates. Therefore, prior to the dive, it would be reasonable to expect a distribution of initial SMB volumes rather than a single definite OW and volume of SMB. The formulation in Eq. 7 is therefore, a simplification.

During descent, the SMB volume will be reduced according to Boyle's law. However, our assumptions do not allow us to decide whether there exists a crushing pressure for the collapse of the SMB as postulated by Yount (1979b).

\section{Limits to the Model: The OW Formulation}

We have used Egi's model of OW, where the role of $\mathrm{CO}_{2}$ is minimized. This is based on the fact that there is no " $\mathrm{CO}_{2}$ window" because $\mathrm{CO}_{2}$ dissolves into $\mathrm{HCO}_{3}$, a weak acid responsible for buffering the blood. The venous $\mathrm{pCO}_{2}$ is considered constant. On this basis, when the diver performs exercise, he increases his tissue metabolism and oxygen consumption, but the mathematical description shows that the OW remains almost unaffected because of the large availability of oxygen in the arterial side. With such a model, the influence of exercise on the tissue OW is small (a few $\mathrm{hPa}$ ) at surface and is negligible in diving conditions up to $2000 \mathrm{hPa}$ of inspired oxygen.

Other definitions of the OW exist depending on the assumptions considered. For instance, Kot et al. (2015) defined the OW using tissue metabolic gases values. This provided a higher estimate of the OW that they called the extended oxygen window.

Van Liew developed an improved OW model by taking into account $\mathrm{CO}_{2}$ variations with exercise (Van Liew et al., 1993) and the influence of $\mathrm{CO}_{2}$ on the oxygen dissociation curve. An increase of the $\mathrm{P}_{\mathrm{v}, \mathrm{CO} 2}$ in Eq. 3 decreases the tissue OW. Consequently, according to governing Eq. 7, it results in a larger volume SMB. A high level of tissue $\mathrm{CO}_{2}$ could be the explanation to the findings of Wilbur et al. who detected, at surface, with a dual frequency ultrasound, a signal consistent with microbubbles after intense exercise on an ergonomic bicycle in a human subject's leg (Wilbur et al., 2010) without any decompression.

Another improvement on the OW calculation was proposed by Walsh et al. who introduced the Michaelis Menten equation to describe the kinetic of oxygen uptake (Walsh et al., 2017). This relation allows computing a local non-linear oxygen consumption and accounts for the fact that different tissues may have different metabolism and therefore different OW's. It could be the way to a more realistic computation of the OW based on a Gaussian distribution of tissue and individual metabolisms.

\section{Decompression: Relation Between Metabolic Gas Pocket and Vascular Gas Emboli Detection}

We refer to the scenario proposed by Arieli and Marmur (2017), where the SMB are activated by decompression and grow until they release as a VGE in the blood stream, after the bubble has reached a critical volume of detachment. If buoyancy is the drive, it means that this critical volume of detachment is independent of the physical shape of the site and is a constant for every diver.

This scenario depends on the dynamic of gas exchanges because the surrounding tissue feeds the SMB, causing growth until it reaches its critical volume. After the first bubble has detached, the site will continue producing bubbles as long as gases will diffuse into it. This suggests that larger initial SMB detach more easily than smaller ones because they can reach this critical volume more rapidly (for the same local gas gradient). Therefore, the initial SMB volume is the first parameter 
controlling VGE once the diver is involved in decompression. This volume in turn depends on the tissue OW according to Eq. 7, which then refers to the diver's metabolism. The interdependence between number of active spots and initial SMB volume could be the reason for the variability in VGE production observed between divers (Balestra, 2014a; Germonpre and Balestra, 2017; Papadopoulou et al., 2018).

\section{Variation of Metabolism: Influence of Age and Fitness on Vascular Gas Emboli Detection}

Metabolism is known to decrease with age. According to Eq. 7, a decreased metabolism, associated to a reduced OW, should yield larger volume initial SMB. This could explain the higher levels of VGE detected in older divers (Carturan et al., 1999; Cialoni et al., 2017). Metabolism therefore introduces age as a first individual characteristic for a diver involved in a decompression.

Metabolism is known to be linearly related to cardiac frequency in rest (Kang et al., 2017). Also, the all-encompassing law of Kleiber has shown a linear relationship between the log of metabolism and the body mass (Kleiber, 1947). These factors suggest that fitness increases the OW (Painter, 2005). According to Eq. 7, an increased metabolism, associated to a higher OW, yield smaller volume SMB. A smaller gas phase takes a longer time to detach and produce fewer circulating bubbles during the decompression time. This would explain the lower levels of VGE observed in fit divers. Metabolism therefore introduces fitness as a second individual characteristic for a diver involved in a decompression.

The action of age and fitness are opposed and independent but can combine in each diver to produce variability. On one hand, an old and sedentary diver should have larger volume of initial SMB than young and fit diver and produce more VGE. On the other hand, an old but fit diver could produce less VGE than a younger but sedentary diver.

In a recent large-scale database analysis, DAN Europe (Cialoni et al., 2017) concluded that only two factors, increased age and BMI, could be related to increased bubble formation. They also noted that neither height or weight separately had any relation to the bubbles. It is tempting to associate BMI to fitness for this divers' populations because it would then provide the confirmation that because age and fitness act on the metabolism, they in turn acts on the OW and finally control the individual part in the bubbling process.

\section{Decreased Metabolism: Influence of Bedrest on Vascular Gas Emboli Detection}

Confirmation of the above analysis is provided by a bedrest experiment recently published by Gennser et al. (2018). Authors used a 35 days' bedrest conditioning to simulate microgravity and then ran several air dives to simulate extra vehicular activity. The dives were performed with divers still in bedrest conditions and were controlled by bubble Doppler monitoring. They concluded that 5 weeks of bedrest significantly increased bubble grades after decompression.
The analysis of this experiment using our model is coherent with the reported results:

- Bedrest conditions are associated to minimal activity and therefore to a minimal metabolism. The consequence is that the initial SMB volume in the divers prior to the dive was maximal according to Eq. 7.

- Then, the lack of exercise reduces vibrations and it is likely that most of the available AHS were populated by SMB.

- After a bedrest, the divers started the dive with a high density of SMB with a maximal volume that favored higher grades of detected VGE.

\section{Vibrations Preconditioning: Influence on Vascular Gas Emboli Detection}

Preconditioning with vibrations produces a local energy release. The energy transferred will move the system to another equilibrium state. In this new state, changes in the internal pressure of the gas phase will modify its volume by either compression or temperature change and/or loss of gasses by diffusion. This will challenge its stability and the SMB may reach the critical volume and detach from its support. The main role of vibrations seems to reduce the number of AHS. The consequence of vibrations alone will be less VGE measured after decompression. However, vibrations do not affect the metabolism and therefore do not change the SMB volume according to Eq. 7.

Divers preconditioning experiments have shown that predive vibrations reduce the number of VGE after decompression (Balestra et al., 2016b). Figure 2 shows VGE counts from this last paper, expressed in number of bubbles detected per heartbeat, at rest and after a leg flexion, for the control dives and the dives with pre-conditioning with vibrations and oxygen.

The protocol of VGE detections, with either Doppler or echocardiography, includes first a measurement at rest and then a measurement after a squat or leg flexions. The flexion generally triggers a rush of circulating bubble provoked by the muscular contractions. Referring to our model, it suggests that this flexion causes SMB to be dislodged from their sites before reaching the critical volume of detachment.

Data in Figure 2 shows that there is no significant difference between rest and flexion after vibration and oxygen preconditioning. For the vibration preconditioning, the explanation could be that SMB have already been dislodged before the dive, so the muscle contraction applies to a smaller number of SMB and produces less VGE (Germonpre and Balestra, 2017).

Data in Figure 2 may allow quantifying this protective effect in term of VGE count reduction, and may make it possible to define simple models and adjust coefficients by data fitting to predict the site density and initial SMB volume of a given diver or group of divers.

\section{Exercise Pre-conditioning: Influence on Vascular Gas Emboli Detection}

Pre-conditioning with exercise is complex because exercise combines several effects. It increases metabolism and shear stress along the endothelium. It also produces a series of metabolic 


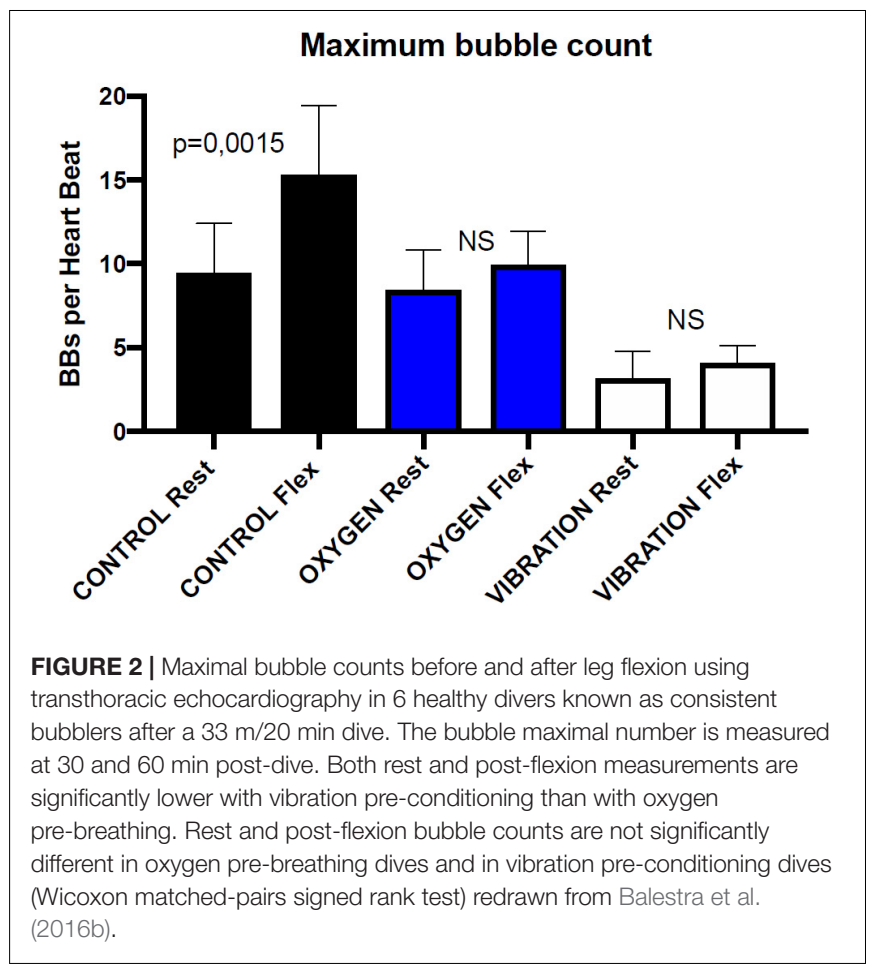

changes: vasodilatation, heat production, $\mathrm{PCO}_{2}$ increase and dehydration. Finally, it causes mechanical vibrations.

This principle of vibrations pre-conditioning from the above paragraph can be extended to exercise pre-conditioning if the metabolic response is ignored. The dose of vibrations obviously varies with the type of activity and this suggests that different exercises may have different efficiencies. For instance, running or mini-trampoline should be more efficient than swimming or cycling. Moreover, considering that the metabolism regenerates the SMB progressively, the frequency of the sport activity becomes important. In fact, the selection of the sport and the frequency of its practice define something we like to call an "healthy life style" (Thompson and Batterham, 2013), which has been suggested to be of importance to reduce diving DCS risk (Carturan et al., 1999, 2002).

\section{Oxygen Breathing Pre-conditioning: Influence on Vascular Gas Emboli Detection}

Divers pre-conditioning experiments have shown that pre-dive oxygen breathing reduces the number of VGE after decompression.

Pre-dive oxygen breathing first involves de-nitrogenation and could protect the diver by reducing his tissue inert gas load before the dive. This protection is relative as it only concerns $790 \mathrm{hPa}$ of surface $\mathrm{PN}_{2}$ compared to a bottom $\mathrm{PN}_{2}$ that could be five times larger.

According to our model, pre-dive oxygen breathing drastically increases the $\mathrm{OW}$ and reduces the SMB volume. Smaller volume SMB will take a longer time to grow and reach the critical volume for detachment and so will result in less VGE (Figure 2). This was confirmed by Van Liew who published that pre-dive oxygen breathing protects rats from decompression bubbles (Van Liew, 1998). However, pre-dive oxygen breathing is not expected to act on the density of SMB sites as vibrations would do (Blatteau et al., 2012).

\section{Combined Vibrations and Oxygen Breathing Pre-conditioning: Influence on Vascular Gas Emboli Detection}

The results (Balestra et al., 2016b; Germonpre and Balestra, 2017) of the combination of vibrations and oxygen breathing in our pre-conditioning experiment showed that in term of post-dive VGE:

- Vibrations better protect the diver than pre-dive oxygen breathing.

- Combination of oxygen breathing before vibration is less efficient than vibrations alone.

Based on our model, these results can be explained by the following contribution of factors:

- On the one hand, vibrations reduce the initial population of SMB but do not change their size. Vibration is efficient in reducing the number of VGE detected after decompression.

- On the other hand, oxygen breathing increases the OW and according to Eq. 7, reduces the SMB volume. Smaller SMB will take a longer time to reach the critical detachment volume and will detach less easily. Pre-dive oxygen breathing is efficient in reducing the number of VGE detected after decompression.

- When combining the two above factors, if oxygen breathing comes first, the SMB will become smaller and more difficult to detach. The consequence will be that the same squeezing action (legs flexion) will detach only the biggest SMB after oxygen breathing than just alone keeping a higher number of VGE. The overall benefit will be less (Figure 2).

Our model suggests that oxygen breathing and vibrations can counteract their effects in this sequence. Alternatively, it proposes that the best pre-conditioning sequence would be to first expose the diver to vibrations to reduce the SMB density and then reduce the volume of the remaining SMB by oxygen breathing. However, this remains to be experimentally tested.

\section{Exercise or Stress During Decompression: Influence on Vascular Gas Emboli Detection}

The situation is different for the previous discussion because change in the SMB population will occur during the dive. In addition, several factors will overlap: metabolism, cardiac function, heat/cold adaptation and exercise. The analysis therefore proceeds with simplification by separating the issues.

With regards to activity during or after the dive, it is understood that light exercise with little metabolic change but 
significant vibrations will release more circulating VGE according to our model predictions. However, two situations may occur.

In a first situation, if the ascent is badly controlled during decompression, or if the decompression table is inadequate, the excess of bubbles dumped into the venous bed might overload the lung and result in arterial bubbles and a higher risk of DCS (Madden and Laden, 2009).

On the other situation, provided the excess bubbles are filtered by the lung, exercise permits evacuating inert gas at a higher rate. Since there is more gas inside a bubble than dissolved in the same volume of blood, this results in a more efficient decompression. Dujic et al. reported that during SCUBA diving, light exercise at the 3 meters stop reduces post-dive VGE (Dujic et al., 2005) and that post-dive exercise induces a more rapid VGE decline (Dujic et al., 2006), indicating a faster decrease of tissue inert gas. Light exercise is therefore beneficial for a decompressing diver. For the same reason, commercial divers in saturation are encouraged to perform a daily session of light exercise during decompression.

With regards to stressful situations during the dive, such as cold or low visibility, they have shown to be statistically related to higher risk of DCS (Cialoni et al., 2017), even if depth and time of the dive were lesser. Stress by itself will trigger a series of physiological reactions such as cardiac frequency and ventilatory rate increase. It will also change the cellular oxygen consumption and metabolic rate as a response to stress and subsequent neuroendocrine reactions. The combined influence of these factors may be as yet not precisely calculable, but will (see Eq. 7) undoubtedly change the population of SMB, their localisation and behavior during decompression.

\section{Metabolism and Diet: Influence on Vascular Gas Emboli Detection}

There is a renewed interest in nutrition in commercial diving to improve divers' performances and eventually reduce their oxidative stress (Deb et al., 2016). It has been suggested that the diver's diet has also a measurable effect on the level of VGE or on the vascular wall compliance which may also influence the SMB elimination (Theunissen et al., 2013, 2015; Valadao et al., 2014; Balestra et al., 2016a).

Metabolism is affected by nutrition. There is a possibility to link nutrition and metabolism through Eq. 7 because Egi's model includes the respiratory quotient in the definition of the OW. This respiratory quotient could be used to link the diver's diet directly to the VGE production.

\section{Implication of Static Metabolic Bubbles on Future Decompression Algorithms}

The link between the presence of VGE and the risk of DCS is not direct. Even though Doppler monitoring has been used as the principal endpoint for the development and validation of most air tables (Spencer, 1976) and mixed gas tables (Nishi, 1991), large scale studies have demonstrated that:

- Vascular gas emboli may be present in any decompression without provoking any symptoms of DCS (so-called silent bubbles).
- There is a large variability among divers in term of VGE production (Papadopoulou et al., 2018).

- It remains that the lower the bubble count (or grade) the lesser the risk of DCS (Nashimoto and Gottoh, 1978; Gardette, 1979; Eftedal et al., 2007).

Linking VGE and the risk of DCS requires establishing a bubble scenario. In 1908, J. S. Haldane stated that "If small bubbles are carried through the lung capillaries and pass, for instance, to a slowly desaturating part of the spinal cord, they will there increase in size and may produce serious blockage of the circulation or direct mechanical damage" (Boycott et al., 1908). This scenario considers intravascular bubbles collected by the venous system and eliminated at the lung level in normal dive conditions. If a bubble passes into the arterial side, it may reach a tissue and causes local ischemia, mechanical damages and inflammation. The filtering capacity of the lung was first studied by Hills (Butler and Hills, 1979). Failure of the lung filter was proposed by James for the onset of CNS and spinal symptoms (James, 1982; James, 1991). Shunting of the lung filter explained the role of a patent foramen ovale (PFO) in the diver's susceptibility to Type II DCS (Moon et al., 1989; Wilmshurst et al., 1989; Balestra and Germonpre, 2016; Lafere et al., 2017). Finally, Hennessy published in 1989 all the physical aspects of the arterial bubbles scenario in a paper that became the foundation of the arterial bubble assumption (Hennessy, 1989).

We have adopted this scenario to link VGE and the risk of DCS:

- The dose of incoming VGE and the lung filtration capacity determine the possibility of arterial bubble occurrence (Butler and Hills, 1979; Ljubkovic et al., 2012).

- The bubbles in the venous system trigger biological reactions with the vascular endothelium that create microparticles. These microparticles can pass in the arterial system and provoke a tissue inflammation similar to the one caused by bubbles (Thom et al., 2013, 2015; Yang et al., 2015).

We therefore consider that arterial bubbles and microparticles combine in the onset of DCS (Arieli and Marmur, 2017). The dose of incoming VGE produced by decompression remains the critical input to the lung filter.

The SMB assumption adds a step in the chain of events triggered by decompression. This intermediate step corresponds to a gas phase, located in the middle of scale, between nanobubbles nucleating at the AHS and micrometric or millimetric VGE dumped into the venous circulation.

Setting an acceptable level of decompression stress in a future algorithm will require defining four additional parameters. The first one will be estimate of the number and size of SMB, based on the diver' individual characteristics. The second parameter will be the critical volume for bubble detachment that will control VGE production. The third will be the lung bubble filtration function that will determine the risk of passing arterial bubbles from the input dose of VGE (volume and number). The fourth one will be a risk function associating the number of arterial bubbles to the risk of DCS symptoms. Further improvements could consider the 
evolution of the number of sites and SMB volumes with repetitive diving and multi-days diving.

It makes it possible to supplement $M$-Values and gradient factors to decompression algorithms based on an initial SMB population adapted to the diver's individual parameters.

The new algorithms will not necessarily produce much different decompression profiles. The current profiles were developed empirically but already provided an acceptable level of risk. Most instances of DCS, however, occur within the limits of these decompression profiles, for reasons as yet unaccounted for. This model could provide a better control of conservatism and offer the possibility to a given diver to select the level of decompression stress he is ready to accept for a given dive.

The pre-existing gas pocket population (SMB) therefore, becomes the diver's main individual characteristic defining the VGE level measured during or after a decompression. It may also contribute to the other dimension of DCS, that is inflammation. Bubbles that detach from their support can strip apart the AHS as observed by Arieli (2017). The AHS may then reduce in size or even disappear after a series of bubbles detachments. Arieli used this possibility to explain divers' acclimatization to intensive and repetitive decompressions. We consider that when VGEs are present, they could generate an inflammatory response because the endothelium would be physically altered by the "rubbing" effect of the VGEs. Causing some damage and retraction of endothelial cells, exposing the endothelial basement membrane to the blood and elicit interaction with inflammatory proteins (Blatteau et al., 2018).

\section{CONCLUSION}

We postulated a pre-existing population of small static bubbles in divers, located preferentially on hydrophobic sites on the endothelial surface, populated and stabilized by tissue metabolism.

These gas pockets are expected to have an intermediate volume set between the nanobubbles initially cavitating at the

\section{REFERENCES}

Arieli, R. (2015). Was the appearance of surfactants in air breathing vertebrates ultimately the cause of decompression sickness and autoimmune disease? Respir. Physiol. Neurobiol. 206, 15-18. doi: 10.1016/j.resp.2014. 11.008

Arieli, R. (2017). Nanobubbles form at active hydrophobic spots on the luminal aspect of blood vessels: consequences for decompression illness in diving and possible implications for autoimmune disease-an overview. Front. Physiol. 8:591. doi: 10.3389/fphys.2017.00591

Arieli, R., Arieli, U., and Marmur, A. (2015). Bubble size on detachment from the luminal aspect of ovine large blood vessels after decompression: the effect of mechanical disturbance. Respir. Physiol. Neurobiol. 216, 1-8. doi: 10.1016/j.resp. 2015.05.001

Arieli, R., and Marmur, A. (2011). Decompression sickness bubbles: are gas micronuclei formed on a flat hydrophobic surface? Respir. Physiol. Neurobiol. 177, 19-23. doi: 10.1016/j.resp.2011.02.013

Arieli, R., and Marmur, A. (2013a). Dynamics of gas micronuclei formed on a flat hydrophobic surface, the predecessors of decompression bubbles. Respir. Physiol. Neurobiol. 185, 647-652. doi: 10.1016/j.resp.2012. 11.020
AHS and the critical volume bubbles that detach from these AHS to become VGE.

We derived a stability equation linking the $\mathrm{OW}$ to the metabolic gas pocket volume without having to define any geometrical configuration.

We tested the assumption against our published experimental data and other relevant papers and found that pre-existing SMB:

- Are consistent with the observations on ex-vivo tissue bubble production by Arieli. This suggests that the level of circulating VGE depends on two parameters: the number of hydrophobic sites and the volume of the metabolic bubbles populating these sites.

- Can explain the results of diver pre-conditioning experiments using exercise, vibrations, oxygen breathing and combined vibration and oxygen breathing.

- Could explain why the individual variations of detected VGE in divers after decompression depend on the density of AHS and therefore seem to be linked to age and fitness.

- Suggest that physical activity type and frequency control this initial metabolic bubble population and therefore the level of VGE produced during a given decompression. In addition to specific individual factors, the diver's "life style" could influence the observed variations in postdecompression VGE.

Our model links these small metabolic gas pockets to VGE measurements and the potential risk of DCS. This model offers two new possibilities for decompression algorithms (1) to adapt decompression to the individual factors of a given diver and (2) to select the level of acceptable decompression stress or DCS risk for a given dive.

\section{AUTHOR CONTRIBUTIONS}

J-PI and SME developed the hypothesis. PG reviewed the medical implications. CB developed the physiological part of the theory.

Arieli, R., and Marmur, A. (2013b). Evolution of bubbles from gas micronuclei formed on the luminal aspect of ovine large blood vessels. Respir. Physiol. Neurobiol. 188, 49-55. doi: 10.1016/j.resp.2013.04.013

Arieli, R., and Marmur, A. (2014). Ex vivo bubble production from ovine large blood vessels: size on detachment and evidence of "active spots". Respir. Physiol. Neurobiol. 200, 110-117. doi: 10.1016/j.resp.2014.05.014

Arieli, R., and Marmur, A. (2017). A biophysical vascular bubble model for devising decompression procedures. Physiol. Rep. 5:e13191. doi: 10.14814/phy2.13191

Arieli, Y., Arieli, R., and Marx, A. (2002). Hyperbaric oxygen may reduce gas bubbles in decompressed prawns by eliminating gas nuclei. J. Appl. Physiol. 92, 2596-2599. doi: 10.1152/japplphysiol.01059.2001

Balestra, C. (2014a). Just say NO to decompression bubbles: is there a real link between nitric oxide and bubble production or reduction in humans? Diving Hyperb. Med. 44, 63-64.

Balestra, C. (2014b). The lymphatic pathway for microbubbles. Diving Hyperb. Med. 44:1.

Balestra, C., Cimino, F., Theunissen, S., Snoeck, T., Provyn, S., Canali, R., et al. (2016a). A red orange extract modulates the vascular response to a recreational dive: a pilot study on the effect of anthocyanins on the physiological consequences of scuba diving. Nat. Prod. Res. 30, 2101-2106. doi: 10.1080/ 14786419.2015.1107062 
Balestra, C., Theunissen, S., Papadopoulou, V., Le Mener, C., Germonpre, P., Guerrero, F., et al. (2016b). Pre-dive whole-body vibration better reduces decompression-induced vascular gas emboli than oxygenation or a combination of both. Front. Physiol. 7:586.

Balestra, C., and Germonpre, P. (2016). Correlation between patent foramen ovale, cerebral "lesions" and neuropsychometric testing in experienced sports divers: does diving damage the brain? Front. Psychol. 7:696.

Behnke, A. R. (1967). "The isobaric (oxygen window) principle of decompression," in Proceedings of the The New Thrust Seaward. Third Marine Technology Society Conference, (San Diego CA: Marine Technology Society).

Blatteau, J.-E., Gaillard, S., De Maistre, S., Richard, S., Louges, P., Gempp, E., et al. (2018). Reduction in the level of plasma mitochondrial DNA in human diving, followed by an increase in the event of an accident. Front. Physiol. 9:1695. doi: $10.3389 /$ fphys.2018.01695

Blatteau, J. E., Gempp, E., Balestra, C., Mets, T., and Germonpre, P. (2008). Predive sauna and venous gas bubbles upon decompression from $400 \mathrm{kPa}$. Aviat. Space Environ. Med. 79, 1100-1105. doi: 10.3357/asem.2377.2008

Blatteau, J. E., Hugon, J., Gempp, E., Castagna, O., Peny, C., and Vallee, N. (2012). Oxygen breathing or recompression during decompression from nitrox dives with a rebreather: effects on intravascular bubble burden and ramifications for decompression profiles. Eur. J. Appl. Physiol. 112, 2257-2265. doi: 10.1007/ s00421-011-2195-6

Blatteau, J. E., Souraud, J. B., Gempp, E., and Boussuges, A. (2006). Gas nuclei, their origin, and their role in bubble formation. Aviat. Space Environ. Med. 77, 1068-1076.

Boycott, A. E., Damant, G. C., and Haldane, J. S. (1908). The prevention of compressed-air illness. J. Hyg. 8, 342-443.

Butler, B. D., and Hills, B. A. (1979). The lung as a filter for microbubbles. J. Appl. Physiol. 47, 537-543. doi: 10.1152/jappl.1979.47.3.537

Carturan, D., Boussuges, A., Burnet, H., Fondarai, J., Vanuxem, P., and Gardette, B. (1999). Circulating venous bubbles in recreational diving: relationships with age, weight, maximal oxygen uptake and body fat percentage. Int. J. Sports Med. 20, 410-414. doi: 10.1055/s-2007-971154

Carturan, D., Boussuges, A., Vanuxem, P., Bar-Hen, A., Burnet, H., and Gardette, B. (2002). Ascent rate, age, maximal oxygen uptake, adiposity, and circulating venous bubbles after diving. J. Appl. Physiol. 93, 1349-1356. doi: 10.1152/ japplphysiol.00723.1999

Castagna, O., Brisswalter, J., Vallee, N., and Blatteau, J. E. (2011). Endurance exercise immediately before sea diving reduces bubble formation in scuba divers. Eur. J. Appl. Physiol. 111, 1047-1054. doi: 10.1007/s00421-010-1723-0

Castagna, O., Gempp, E., and Blatteau, J. E. (2009). Pre-dive normobaric oxygen reduces bubble formation in scuba divers. Eur. J. Appl. Physiol. 106, 167-172. doi: 10.1007/s00421-009-1003-z

Chappell, M. A., and Payne, S. J. (2006). A physiological model of the release of gas bubbles from crevices under decompression. Respir. Physiol. Neurobiol. 153, 166-180. doi: 10.1016/j.resp.2005.10.006

Chappell, M. A., and Payne, S. J. (2007). The effect of cavity geometry on the nucleation of bubbles from cavities. J. Acoust. Soc. Am. 121, 853-862. doi: $10.1121 / 1.2404629$

Cialoni, D., Pieri, M., Balestra, C., and Marroni, A. (2017). Dive risk factors, gas bubble formation, and decompression illness in recreational SCUBA diving: analysis of DAN Europe DSL data base. Front. Psychol. 8:1587. doi: 10.3389/ fpsyg.2017.01587

Deb, S. K., Swinton, P. A., and Dolan, E. (2016). Nutritional considerations during prolonged exposure to a confined, hyperbaric, hyperoxic environment: recommendations for saturation divers. Extrem. Physiol. Med. 5:1. doi: 10.1186/ s13728-015-0042-9

Doolette, D. J. (2019). Gas micronuclei that underlie decompression bubbles and decompression sickness have not been identified. Diving Hyperb. Med. 49:64. doi: $10.28920 / \mathrm{dhm} 49.1 .64$

Dujic, Z., Duplancic, D., Marinovic-Terzic, I., Bakovic, D., Ivancev, V., Valic, Z., et al. (2004). Aerobic exercise before diving reduces venous gas bubble formation in humans. J. Physiol. 555, 637-642. doi: 10.1113/jphysiol.2003. 059360

Dujic, Z., Obad, A., Palada, I., Ivancev, V., and Valic, Z. (2006). Venous bubble count declines during strenuous exercise after an open sea dive to $30 \mathrm{~m}$. Aviat. Space Environ. Med. 77, 592-596.

Dujic, Z., Palada, I., Obad, A., Duplancic, D., Bakovic, D., and Valic, Z. (2005). Exercise during a 3-min decompression stop reduces postdive venous gas bubbles. Med. Sci. Sports Exerc. 37, 1319-1323. doi: 10.1249/01.mss. 0000174892.27331.ce

Eftedal, O. S., Tjelmeland, H., and Brubakk, A. O. (2007). Validation of decompression procedures based on detection of venous gas bubbles: a Bayesian approach. Aviat. Space Environ. Med. 78, 94-99.

Egi, S. M. (1994). "Estimation of oxygen window during and after altitude exposures," in Proceedings of the 20 th Annual Meeting of European Underwater and Biomedical Society, (Heraklion: European Underwater and Biomedical Society), 135-139.

Evans, A., and Walder, D. N. (1969). Significance of gas micronuclei in the aetiology of decompression sickness. Nature 222, 251-252. doi: 10.1038/222251a0

Foster, P. P., Conkin, J., Powell, M. R., Waligora, J. M., and Chhikara, R. S. (1998). Role of metabolic gases in bubble formation during hypobaric exposures. J. Appl. Physiol. 84, 1088-1095. doi: 10.1152/jappl.1998.84.3.1088

Gardette, B. (1979). Correlation between decompression sickness and circulating bubbles in 232 divers. Undersea Biomed. Res. 6, 99-107.

Gennser, M., Blogg, S. L., Eiken, O., and Mekjavic, I. B. (2018). Indices of increased decompression stress following long-term bed rest. Front. Physiol. 9:442. doi: $10.3389 /$ fphys.2018.00442

Germonpre, P., and Balestra, C. (2017). Preconditioning to reduce decompression stress in scuba divers. Aerosp. Med. Hum. Perform. 88, 114-120. doi: 10.3357/ AMHP.4642.2017

Germonpre, P., Papadopoulou, V., Hemelryck, W., Obeid, G., Lafere, P., Eckersley, R. J., et al. (2014). The use of portable 2D echocardiography and 'frame-based' bubble counting as a tool to evaluate diving decompression stress. Diving Hyperb. Med. 44, 5-13.

Germonpre, P., Pontier, J. M., Gempp, E., Blatteau, J. E., Deneweth, S., Lafere, P., et al. (2009). Pre-dive vibration effect on bubble formation after a 30-m dive requiring a decompression stop. Aviat. Space Environ. Med. 80, 1044-1048. doi: $10.3357 /$ asem.2588.2010

Goldman, S. (2010). Free energy wells for small gas bubbles in soft deformable materials. J. Chem. Phys. 132:164509. doi: 10.1063/1.3394940

Harvey, E. N., Barnes, D. K., Mcelroy, W. D., Whiteley, A. H., Pease, D. C., and Cooper, K. W. (1944). Bubble formation in animal. Physical factors. J. Cell. Comp. Physiol. 24, 1-22.

Hennessy, T. R. (1989). "On the site of origin, evolution and effects of dexompression microbubbles," in Proceedings of the International symposium on supersaturation and bubble formation in fluids and organisms, Trondheim.

Hills, B. A. (1967). Decompression sickness: a study of cavitation at the liquidliquid interface. Aerosp. Med. 38, 814-817.

Hills, B. A. (1970). Limited supersaturation versus phase equilibration in predicting the occurrence of decompression sickness. Clin. Sci. 38, 251-267. doi: 10.1042/ cs0380251

Hills, B. A. (1992). A hydrophobic oligolamellar lining to the vascular lumen in some organs. Undersea Biomed. Res. 19, 107-120.

Hills, B. A., and LeMessurier, D. H. (1969). Unsaturation in living tissue relative to the pressure and composition of inhaled gas and its significance in decompression theory. Clin. Sci. 36, 185-195.

Hugon, J. (2014). Decompression models: review, relevance and validation capabilities. Undersea Hyperb. Med. 41, 531-556.

Hugon, J., Barthelemy, L., Rostain, J. C., and Gardette, B. (2009). The pathway to drive decompression microbubbles from the tissues to the blood and the lymphatic system as a part of this transfer. Undersea Hyperb. Med. 36, 223-236.

Ishiyama, A. (1983). Analysis of gas composition of intravascular bubbles produced by decompression. Bull. Tokyo Med. Dent. Univ. 30, 25-35.

James, P. B. (1982). "The size distribution of gas emboli arising during decompression. A review of the concept of critical diameter of gas emboli," in Proceedings of the XIIIth Annual Congress of the EUBS, (Lübec: dragerwerke).

James, P. B. (1991). "Decompression sickness," in Clinical Neurology, eds M. Swash and J. Oxbury (London: Churchill Livingstone).

Kang, S. J., Ha, G. C., and Ko, K. J. (2017). Association between resting heart rate, metabolic syndrome and cardiorepiratory fitness in Korean male adult. J. Exerc. Sci. Fit. 15, 27-31. doi: 10.1016/j.jesf.2017.06.001

Kleiber, M. (1947). Body size and metabolic rate. Physiol. Rev. 27, 511-541. doi: 10.1152/physrev.1947.27.4.511

Kot, J., Sicko, Z., and Doboszynski, T. (2015). The extended oxygen window concept for programming saturation decompressions using air and nitrox. PLoS One 10:e0130835. doi: 10.1371/journal.pone.0130835 
Lafere, P., Balestra, C., Caers, D., and Germonpre, P. (2017). Patent foramen ovale (PFO), personality traits, and iterative decompression sickness. retrospective analysis of 209 cases. Front. Psychol. 8:1328. doi: 10.3389/fpsyg.2017.01328

Ljubkovic, M., Zanchi, J., Breskovic, T., Marinovic, J., Lojpur, M., and Dujic, Z. (2012). Determinants of arterial gas embolism after scuba diving. J. Appl. Physiol. 112, 91-95. doi: 10.1152/japplphysiol.00943.2011

Madden, L. A., and Laden, G. (2009). Gas bubbles may not be the underlying cause of decompression illness - the at-depth endothelial dysfunction hypothesis. Med. Hypoth. 72, 389-392. doi: 10.1016/j.mehy.2008.11.022

Moon, R. E., Camporesi, E. M., and Kisslo, J. A. (1989). Patent foramen ovale and decompression sickness in divers. Lancet 1, 513-514. doi: 10.1016/s01406736(89)90064-0

Nashimoto, I., and Gottoh, Y. (1978). "Relationship between precordial doppler ultrasound records and decompression sickness," in Proceedings of the 6th Symposium of Underwater Physiology, eds M. C. W. Shilling and M. W. Beckett (Bethesda, MD: Federation of American Societies for Experimental Biology), 497-501.

Nishi, R. Y. (1991). Development of surface decompression tables for heliumoxygen diving for depths to $100 \mathrm{msw}$. Undersea Biomed. Res. 18(Suppl.), 66-67.

Nishi, R. Y., Brubakk, A. O., and Eftedal, O. S. (2003). "Bubble detection," in Bennett and Elliott's Physiology and Medicine of Diving, 5th Edn, eds A. O. Brubakk and T. S. Neuman (Livonia, MI: Saunders).

Ohgaki, K., Khanh, N. Q., Joden, Y., Tsuji, A., and Nakagawa, T. (2010). Physicochemical approach to nanobubble solutions. Chem. Eng. Sci. 65, 12961300. doi: 10.1016/j.ces.2009.10.003

Painter, P. R. (2005). Allometric scaling of the maximum metabolic rate of mammals: oxygen transport from the lungs to the heart is a limiting step. Theor. Biol. Med. Model. 2:31.

Papadopoulou, V., Balestra, C., Theunissen, S., Germonpré, P., Obeid, G., Boutros, A., et al. (2017). "Can current contrast mode echocardiography help estimate bubble population dynamics post-dive," in Proceedings of the $43 \mathrm{rd}$ Annual scientific Meeting of the European Underwater \& Baromedical Society, (Ravenna: European Underwater \& Baromedical Society).

Papadopoulou, V., Eckersley, R. J., Balestra, C., Karapantsios, T. D., and Tang, M. X. (2013). A critical review of physiological bubble formation in hyperbaric decompression. Adv. Colloid Interface Sci. 19, 22-30. doi: 10.1016/j.cis.2013. 02.002

Papadopoulou, V., Evgenidis, S., Eckersley, R. J., Mesimeris, T., Balestra, C., Kostoglou, M., et al. (2015). Decompression induced bubble dynamics on ex vivo fat and muscle tissue surfaces with a new experimental set up. Colloids Surf. B Biointerfaces 129, 121-129. doi: 10.1016/j.colsurfb.2015.03.027

Papadopoulou, V., Germonpre, P., Cosgrove, D., Eckersley, R. J., Dayton, P. A., Obeid, G., et al. (2018). Variability in circulating gas emboli after a same scuba diving exposure. Eur. J. Appl. Physiol. 118, 1255-1264. doi: 10.1007/s00421018-3854-7

Papadopoulou, V., Tang, M. X., Balestra, C., Eckersley, R. J., and Karapantsios, T. D. (2014). Circulatory bubble dynamics: from physical to biological aspects. $A d v$. Colloid Interface Sci. 206, 239-249. doi: 10.1016/j.cis.2014.01.017

Spencer, M. P. (1976). Decompression limits for compressed air determined by ultrasonically detected blood bubbles. J. Appl. Physiol. 40, 229-235. doi: 10. 1152/jappl.1976.40.2.229

Spisni, E., Marabotti, C., De Fazio, L., Valerii, M. C., Cavazza, E., Brambilla, S., et al. (2017). A comparative evaluation of two decompression procedures for technical diving using inflammatory responses: compartmental versus ratio deco. Diving Hyperb. Med. 47, 9-16. doi: 10.28920/dhm47.1.9-16

Theunissen, S., Balestra, C., Boutros, A., De Bels, D., Guerrero, F., and Germonpre, P. (2015). The effect of pre-dive ingestion of dark chocolate on endothelial function after a scuba dive. Diving Hyperb. Med. 45, 4-9.

Theunissen, S., Schumacker, J., Guerrero, F., Tillmans, F., Boutros, A., Lambrechts, K., et al. (2013). Dark chocolate reduces endothelial dysfunction after successive breath-hold dives in cool water. Eur. J. Appl. Physiol. 113, 2967-2975. doi: 10.1007/s00421-013-2732-6

Thom, S. R., Bennett, M., Banham, N. D., Chin, W., Blake, D. F., Rosen, A., et al. (2015). Association of microparticles and neutrophil activation with decompression sickness. J. Appl. Physiol. 119, 427-434. doi: 10.1152/ japplphysiol.00380.2015

Thom, S. R., Milovanova, T. N., Bogush, M., Yang, M., Bhopale, V. M., Pollock, N. W., et al. (2013). Bubbles, microparticles, and neutrophil activation: changes with exercise level and breathing gas during open-water SCUBA diving. J. Appl. Physiol. 114, 1396-1405. doi: 10.1152/japplphysiol.00106.2013

Thom, S. R., Yang, M., Bhopale, V. M., Huang, S., and Milovanova, T. N. (2011). Microparticles initiate decompression-induced neutrophil activation and subsequent vascular injuries. J. Appl. Physiol. 110, 340-351. doi: 10.1152/ japplphysiol.00811.2010

Thompson, D., and Batterham, A. M. (2013). Towards integrated physical activity profiling. PLoS One 8:e56427. doi: 10.1371/journal.pone.0056427

Tikuisis, P. (1986). Modeling the observations of invivo bubble formation with hydrophobic crevices. Undersea Biomed. Res. 13, 165-180.

Turmine, M., Mayaffre, A., and Letellier, P. (2004). Nonextensive approach to thermodynamics: analysis and suggestions, and application to chemical reactivity. J. Phys. Chem. B 108, 18980-18987. doi: 10.1021/jp046849\%2B

Valadao, J. M., Vigilante, J. A., Digeorge, N. W., O'connor, S. E., Bear, A., Kenyon, J., et al. (2014). Ketogenic diet for high partial pressure oxygen diving. Undersea Hyperb. Med. 41, 331-335.

Van Liew, H. D. (1962). Tissue pO2 and pCO2 estimation with rat subcutaneous gas pockets. J. Appl. Physiol. 17, 851-855. doi: 10.1152/jappl.1962.17.6.851

Van Liew, H. D. (1998). Evidence that Breathing of Oxygen Inactivates Precursors of Decompression Bubbles. North Palm Beach, FL: Undersea and Hyperbaric Medical Society.

Van Liew, H. D., Conkin, J., and Burkkard, M. E. (1993). The oxygen window and decompression bubbles. Estimates and significance. Aviat. Space Environ. Med. $64,859-865$.

Van Liew, H. D., and Raychaudhuri, S. (1997). Stabilized bubbles in the body: pressure-radius relationships and the limits to stabilization. J. Appl. Physiol. 82, 2045-2053. doi: 10.1152/jappl.1997.82.6.2045

Vann, R. D., Grimstad, J., and Nielsen, C. H. (1980). Evidence for gas nuclei in decompressed rats. Undersea Biomed. Res. 7, 107-112.

Walsh, C., Ovenden, N., Stride, E., and Cheema, U. (2017). Quantification of cell-bubble interactions in a 3D engineered tissue phantom. Sci. Rep. 7:6331. doi: 10.1038/s41598-017-06678-y

Wienke, B., and O'leary, T. (2018). On bubble regeneration and broadening with implications for decompression protocols. J. Appl. Biotechnol. Bioeng. 5, 193-200.

Wilbur, J. C., Phillips, S. D., Donoghue, T. G., Alvarenga, D. L., Knaus, D. A., Magari, P. J., et al. (2010). Signals consistent with microbubbles detected in legs of normal human subjects after exercise. J. Appl. Physiol. 108, 240-244. doi: 10.1152/japplphysiol.00615.2009

Wilmshurst, P. T., Byrne, J. C., and Webb-Peploe, M. M. (1989). Relation between interatrial shunts and decompression sickness in divers. Lancet 2, 1302-1306. doi: 10.1016/s0140-6736(89)91911-9

Wisloff, U., Richardson, R. S., and Brubakk, A. O. (2004). Exercise and nitric oxide prevent bubble formation: a novel approach to the prevention of decompression sickness? J. Physiol. 555, 825-829. doi: 10.1113/jphysiol.2003.055467

Yang, M., Barak, O. F., Dujic, Z., Madden, D., Bhopale, V. M., Bhullar, J., et al. (2015). Ascorbic acid supplementation diminishes microparticle elevations and neutrophil activation following SCUBA diving. Am. J. Physiol. Regul. Integr. Comp. Physiol. 309, R338-R344. doi: 10.1152/ajpregu.00155.2015

Yount, D. E. (1979a). Application of a bubble formation model to decompression sickness in rats and humans. Aviat. Space Environ. Med. 50, 44-50.

Yount, D. E. (1979b). Skins of varying permeability: a stabilization mechanism for gas cavitation nuclei. J. Acoust. Soc. Am.65, 1429-1439. doi: 10.1121/1.382930

Conflict of Interest Statement: J-PI was employed by company Divetech.

The remaining authors declare that the research was conducted in the absence of any commercial or financial relationships that could be construed as a potential conflict of interest.

The reviewer DC declared a shared affiliation, though no other collaboration, with one of the authors SME to the handling Editor.

Copyright $\odot 2019$ Imbert, Egi, Germonpré and Balestra. This is an open-access article distributed under the terms of the Creative Commons Attribution License (CC BY). The use, distribution or reproduction in other forums is permitted, provided the original author(s) and the copyright owner(s) are credited and that the original publication in this journal is cited, in accordance with accepted academic practice. No use, distribution or reproduction is permitted which does not comply with these terms. 